\title{
Access to mammography screening in a large urban population: a multi-level analysis
}

\author{
Stephen C. Meersman • Nancy Breen · \\ Linda W. Pickle $\cdot$ Helen I. Meissner • \\ Paul Simon
}

Received: 28 October 2008/ Accepted: 26 May 2009/Published online: 20 June 2009

(c) The Author(s) 2009. This article is published with open access at Springerlink.com

\begin{abstract}
Objective To understand area-based sociodemographics, physician and medical practice characteristics, and community indicators associated with mammography use in Los Angeles County. An earlier multi-level analysis by Gumpertz et al. found that distance to the nearest mammography facility helped explain the higher proportion of Latinas diagnosed with late stage breast cancer compared with nonLatina Whites in Los Angeles County. Our study examined whether Latinas also have lower rates of mammography use. Methods We used a multi-level spatial modeling approach to examine individual and community level associations with mammography use among a diverse group of women aged 40-84 years in Los Angeles County. To build our
\end{abstract}

S. C. Meersman $(\bowtie)$

Surveillance Research Program, Division of Cancer Control and Population Sciences, National Cancer Institute, 6116 Executive Blvd., Suite 504, Rockville, MD 20892, USA

e-mail: meersmas@mail.nih.gov

N. Breen

Applied Research Program, Division of Cancer Control and Population Sciences, National Cancer Institute, 6116 Executive Blvd., Suite 504, Rockville, MD 20892, USA

L. W. Pickle

StatNet Consulting LLC, Laytonsville, MD, USA

L. W. Pickle

The Pennsylvania State University, University Park, PA, USA

H. I. Meissner

Office of Behavioral and Social Sciences Research, NIH,

Bethesda, MD, USA

P. Simon

Los Angeles County Department of Public Health, Los Angeles, CA, USA multi-level spatial data set, we integrated five data sources: (1) 2001 California Health Interview Survey (CHIS) data, (2) 2001 Food and Drug Administration (FDA) certified mammography facility data, (3) 2003 LA Transit Authority data, (4) 2000 US Decennial Census data, and (5) 2001 Community Tracking Study (CTS) Physician's Survey data.

Results Our study confirmed for Los Angeles County many associations for mammography use found in other locations. An unexpected finding was that women with limited English proficiency (predominantly Latina) were significantly more likely to have had a recent mammogram than English-proficient women. We also found that, after controlling for other factors, mammography use was higher in neighborhoods with a greater density of mammography facilities.

Conclusion Women with limited English proficiency were especially likely to report recent mammography in Los Angeles. This unexpected finding suggests that the intensive Spanish-language outreach program conducted by the Every Woman Counts (EWC) Program in lowincome Latina communities in Los Angeles has been effective. Our study highlights the success of this targeted community-based outreach conducted between 1999 and 2001. These are the same populations that Gumpertz et al. identified as needing intervention. It would be useful to conduct another study of late-stage diagnosis in Los Angeles County to ascertain whether increased rates of mammography have also led to less late-stage diagnosis among Latinas in the neighborhoods where they are concentrated in Los Angeles.

Keywords Breast neoplasms - Mammography · Healthcare disparities - Inequalities · GIS .

Logistic models · Socioeconomic factors .

Multi-level spatial models 


\section{Introduction}

Regular mammography use in the United States involves repeated interactions by women with health care providers. These interactions are mediated by health care markets within the broader social and economic environment. Even though these interactions take place in specific geographic locations in a particular social and economic environment, research has not adequately explored how specific geographic, social and economic environments influence mammography use.

An extensive body of research elucidating the determinants of mammography use has largely focused on correlates associated with adherence to recommended screening intervals that can be measured by self-report [1-3]. These include sociodemographic characteristics such as income, education, and age [4-6]; psychosocial influences such as culture, risk perception, and social support [7-11]; and health care access determinants such as provider recommendation and health insurance status $[12,13]$.

In this study, we used a multi-level spatial analysis to investigate how place of residence, density or proximity to one or more mammography facilities, and other community level variables can help us better understand patterns of mammography use in Los Angeles County. An earlier multi-level analysis by Gumpertz et al. found that the higher proportion of Latinas diagnosed with late stage breast cancer compared with non-Latina Whites in Los Angeles County could be explained by differences in tumor biology, sociodemographic and neighborhood characteristics, and distance to the nearest mammography facility [14]. Other investigations have shown that place of residence influences health outcomes [15, 16], including stage of cancer diagnosis [17].

In recent years, multi-level analytic methods have been employed to simultaneously distinguish the effects of arealevel and individual-level factors on a range of health outcomes [14, 18-20]. Multi-level approaches consistently have found associations between contextual variables and health outcomes above and beyond individual characteristics [21-23]. Because contextual and individual factors interact with one another [24], addressing the interplay of these factors, rather than focusing exclusively on a single factor, can enhance efforts to promote mammography. In this paper, we examine a range of multi-level factors that may influence mammography in Los Angeles County.

\section{Methods}

Conceptual framework and data sources

To conceptualize on-schedule mammography screening, we adapted a multi-level systems framework that integrates individual, practice, and community variables [25]. The three levels of our framework are: (A) Practice setting/ individual level which includes (1) individual patient characteristics, (2) clinician or provider team characteristics, and (3) encounters between doctor and patient; (B) Plan or medical group level which includes policies that can affect delivery of services, such as accepting patients without health insurance, and (C) Social and economic context/community level which includes factors that impact access and availability of mammography, such as the density of facilities or community poverty level.

We linked five data sets to test our systems framework. Our systems framework and geographic nesting structure is shown in Figure 1. The five nested data sets correspond to the three levels in our framework: the practice setting/ individual level, the plan level, and the social and economic context or community level. In order to determine the most appropriate geographic aggregation for each data set, we tested three geographic units: the Census Tract, Health District (HD), and Service Planning Area (SPA). These latter two geographic units are specific to Los Angeles County and were developed for purposes of health and social service planning and service distribution. The 26 HDs and 8 SPAs reflect the health care needs of local communities in LA County.

Individual-level data were obtained from the California Health Interview Survey (CHIS). CHIS, conducted every 2 years, is a random-digit-dial (RDD) telephone survey of adults, adolescents, and children from all California counties. The first survey, conducted in 2001 in five languages, collected information from more than 55,000 households statewide. CHIS is the largest state health survey, one of the largest health surveys in the United States, and one of the few to report county and community level information. By providing information on many racial and ethnic groups and local-level information, CHIS gives health planners, policy makers, county governments, advocacy groups, and communities a detailed picture of the health and health care needs facing California's diverse population. The CHIS conducts multi-language interviews to accommodate the state's large immigrant population. Geocoding provides the opportunity to link CHIS to other data and to perform spatial analysis. Los Angeles was the only county fully geocoded in 2001 CHIS.

Our study sample includes women in Los Angeles County aged 40-84 years who responded to the 2001 CHIS. Geocoding to latitude/longitude was successful for approximately $70 \%$ of the respondents based on the nearest street intersection to their residence, information they provided in the interview. Remaining respondents were geocoded to ZIP Code centroids. All geocoding was done using ESRI's ArcMap software, supplemented by manual lookup. Twenty respondents had neither a complete 
Fig. 1 Combined conceptual framework and geographic nesting structure of data
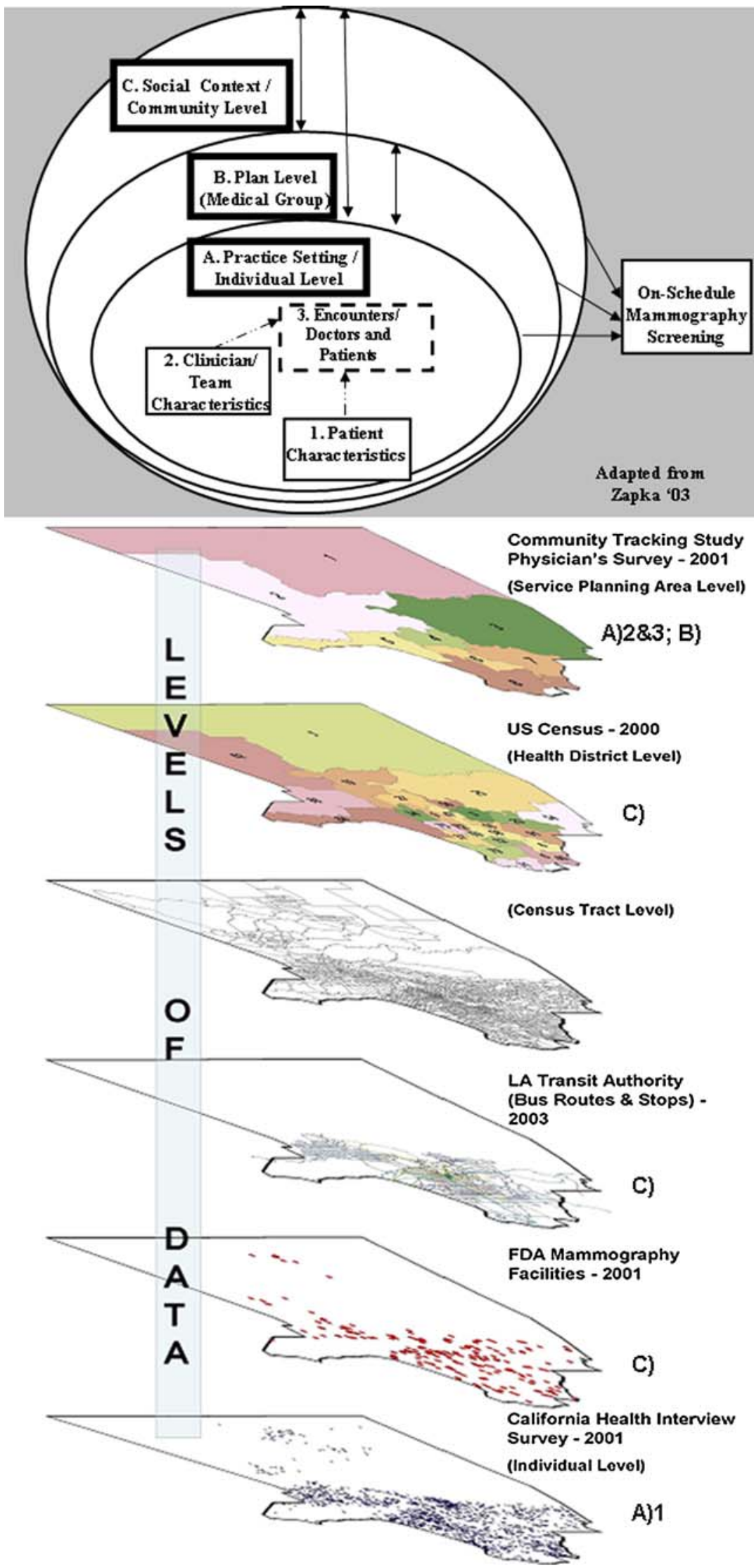
address nor ZIP Code information and were deleted. The resulting study sample of 4,249 women ages 40-84 was linked to the other data sets. All variables tested, regardless of whether we included them in our final models, are shown in the complete variable list in Table 1. Only the variables included in the logistic regression model are presented in Table 2, which also shows the distribution of individual-level CHIS respondent sample characteristics.

In the paragraphs below, we describe the data sets used to measure each level of our conceptual framework, the variables we considered, and the variables included in the best fitting statistical models.

Practice setting/individual level: The practice setting is measured as a relationship between the patient and the clinician or team of clinicians. Different practice settings are associated in the literature with different screening practices [25-32]. 1. Patient characteristics: women sampled in the 2001 California Health Interview Survey (CHIS) aged 40-84 from Los Angeles County were used to estimate on-schedule mammography by patient sociodemographics. All geographic data are linked to this sample of women. We examined information on race/ethnicity, age, education, income, marital status, and English language proficiency as well as indicators of health and health care access. 2. Clinician/team characteristics: to measure practice and personal characteristics of clinicians, we used the 2001 Community Tracking Study (CTS) Physician's Survey. The CTS includes sixty sites nationally (51 metropolitan areas and 9 nonmetropolitan areas) that were randomly selected to form the core of the survey and to be representative of the nation.

Los Angeles County was randomly selected as one site and is used in the present analysis. The CTS Physician survey data were sampled at the ZIP Code level and includes responses from 220 clinicians. The physician sample did not have adequate power at the Zip Code level so responses were aggregated to the SPA level using a crosswalk file. Only aggregate CTS Physician Survey data at the SPA level were large enough to have adequate statistical power. We linked the SPA CTS physician data to CHIS respondent data. See Table 1 (total variable list) for the list of provider variables that we considered.

3. Encounters/interactions between doctor and patients: Similar to those of patients, the sociodemographic characteristics of individual clinicians and medical groups as well as the medical plan structure within which they work, may facilitate or impede regular screening. We created two indices from the CTS data to characterize doctor and patient interactions: ability to provide quality care to patients and ease of quality referrals.

Plan or medical group level: As outlined earlier, the CTS physician survey data were tested for factors that could influence mammography use in a given geographic area. These included the clinician or team characteristics, the reported interactions of providers with patients at the plan or medical group level and the percentage of medical practice revenue obtained from various types of reimbursement. The percentage of practices that did not accept MediCAL (California's Medicaid program) was used as an indicator of access to care for low-income women at the plan or medical group level.

Social and economic context/community level: We used data on location of mammography facility, bus and rail stop locations, and neighborhood sociodemographic characteristics to measure the social and economic context at the community levels. These measures were obtained from the FDA Certified Mammography Facility Address data base [33], the LA County Metro Transit Authority Route and Stop database [34], and the US Census for Census tracts [35] and were linked to the individual-level CHIS respondent data to examine their association with access to on-schedule mammography screening.

Measuring geographic access

A measure of special interest to us was geographic access to mammography. Precise address information for FDA certified mammography facilities from 2001 was used to create two measures potentially associated with access to mammography screening by using a geographic information system (GIS). The first measure is the Euclidean, or straight-line distance from the respondent's address (or Zip Code centroid) to the nearest facility. For the second, we created an alternative measure that indicates the density of facilities within a limited circular buffer immediately surrounding the respondent's address. These methods of aggregation have been previously referred to as "personcentered" because the respondents' address determines the center of the buffer and the density of facilities surrounding a respondent's residence [36].

Measuring the distance to the facility nearest to a respondent's address provides an estimate of the closest facility while density measures the number of facilities within a designated geographic area. The density of facilities surrounding a respondent's residence also represents greater choice of facilities. Because distance and density are conceptually different aspects of potential access and may yield different results, we examined both measures.

To decide which measure to use, we first calculated density. A GIS was used to calculate 1, 2, 3, 4, 5, and 10mile buffers immediately surrounding a woman's place of residence. We then computed the number of FDA certified mammography facilities within each buffer. Statistical modeling was used to determine the single most appropriate buffer distance because overlapping buffers were collinear. After trying a priori distance cutoffs for each 
Table 1 Total variable list prior to statistical selection coded to data sources and conceptual framework Los Angeles county women aged $40-84$ who received a mammogram within past 2 years

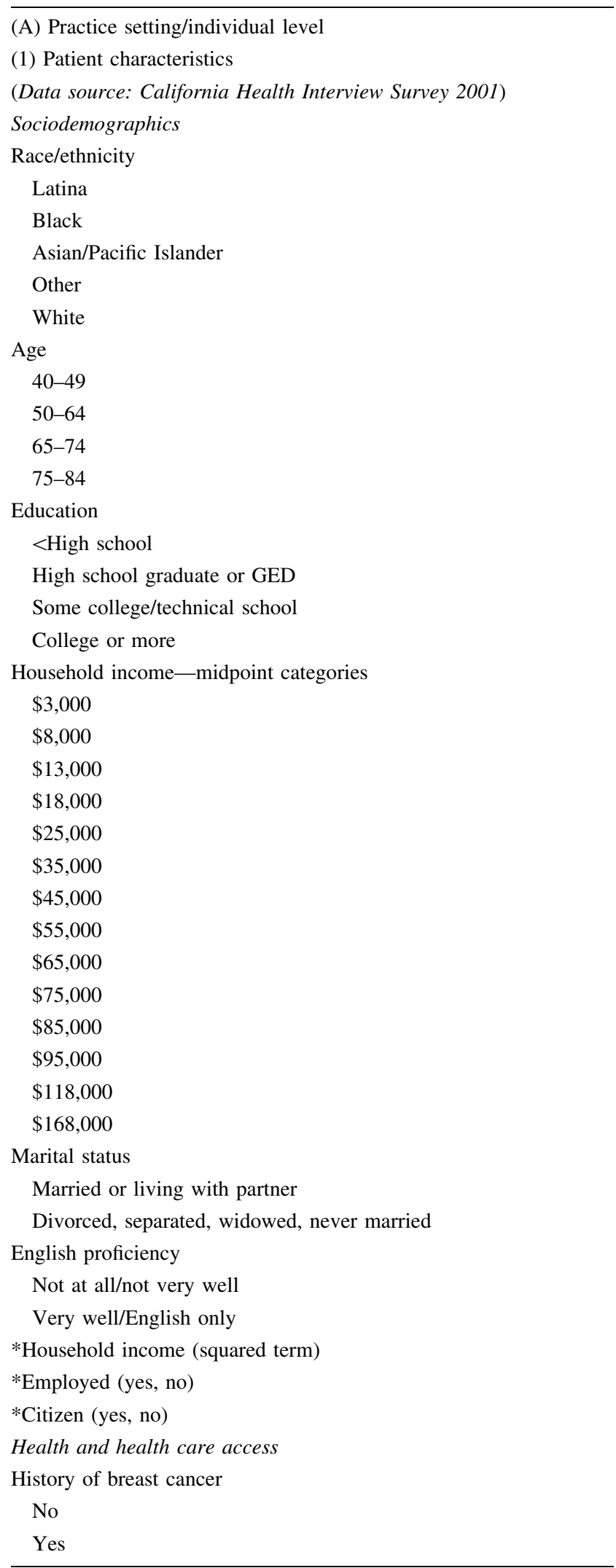

Table 1 continued

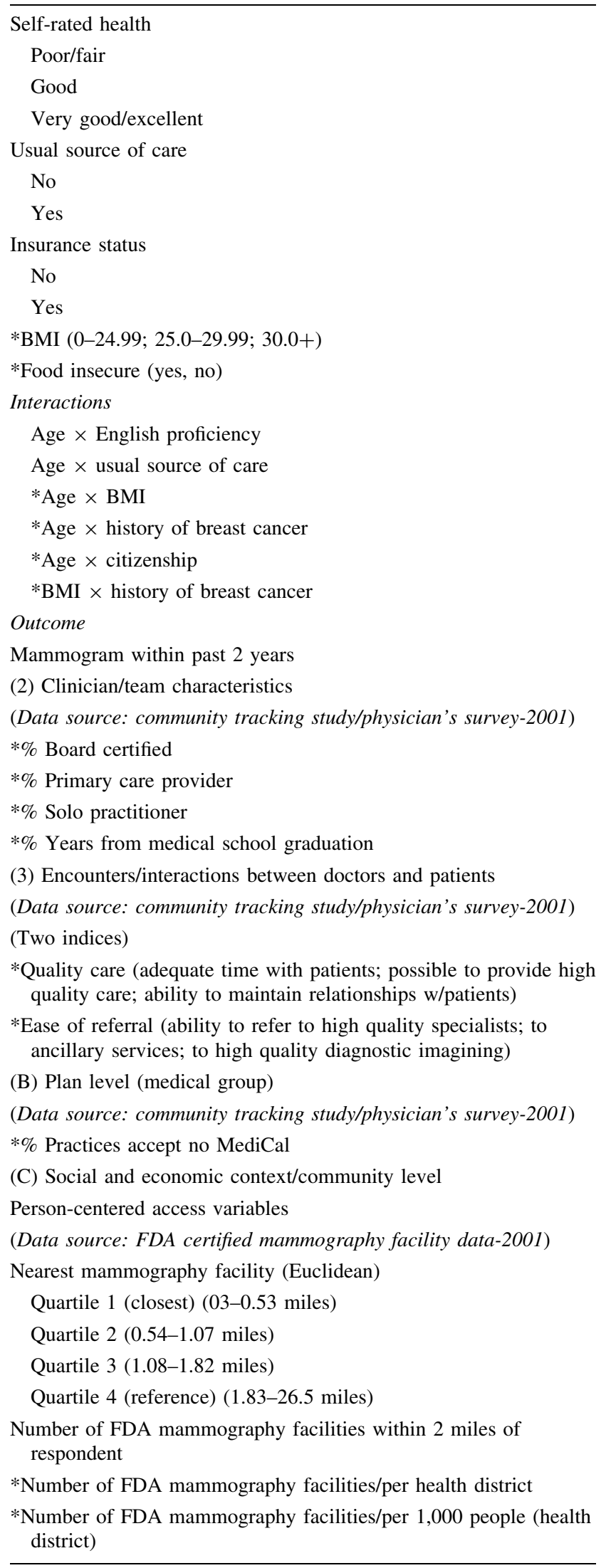


Table 1 continued

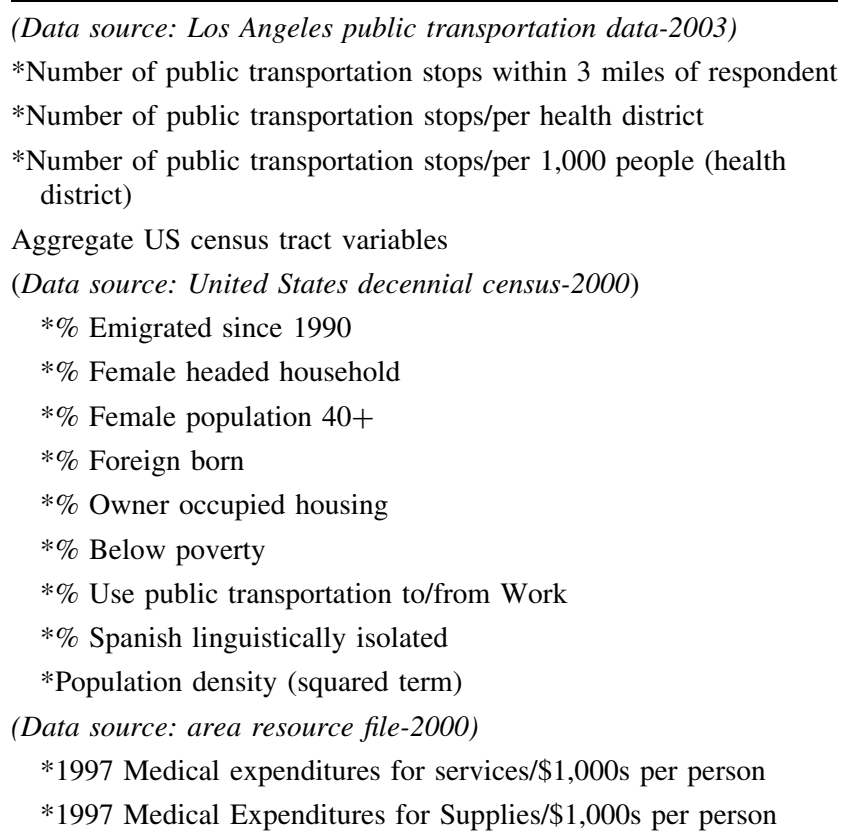

* Variables stepped out of the models based on statistical criteria described in text

buffer density variable, we selected the model with the 2mile buffer of mammography facility density because it had the lowest Akaike Information Criterion (AIC) [37], indicating the best statistical fit.

Los Angeles County Metro Transit Authority (LAMTA) bus route and rail line stop data were computed as the combination of all routes and individual stops for each transit location per day. Bus and rail data from 2003 were manipulated similar to the person-centered density variable for mammography facilities described earlier. A 3-mile buffer distance was selected for inclusion in the model using the lowest AIC.

We tested aggregate information on demographic and socioeconomic data from the 2000 US Decennial Census (Sample File 3) at each geographic unit described earlier: the Census Tract, HD, and SPA. Based on the distribution of CHIS respondents in each geographic area and the statistical selection criteria outline below, we aggregated Census information to the HD level. A detailed list of these variables is included in Table 1.

\section{Statistical analysis}

We used a combination of theoretical and statistical procedures to select and specify variables for our models from the list of variables shown in Table 1. Several covariates considered for inclusion on theoretical grounds were deleted due to collinearity. For the remaining continuous covariates, we determined appropriate transformations using logit plots. If the association between mammography use (as measured by the logit, $\ln (p /(1+p))$, where $p$ is the probability of mammography use) and a covariate was linear then the covariate was included in the model as a continuous variable. If a simple transformation such as the square root of a covariate did not achieve linearity, then the covariate was categorized. Income (in thousands of dollars), age, and body mass index (BMI) were categorized. Age was categorized into four groups: 40-49, 50-64, 6574 , and 75-84 years. We used US Preventive Services Task Force (USPSTFS) and Medicare eligibility guidelines to determine appropriate categorizations for age. BMI was grouped into three conventional categories: underweight (0-18.49), normal weight (18.5-24.99), and overweight $(25.0+)$. The density of FDA certified mammography facilities within 2 miles of each respondent's home was converted into quartiles $(0-1,2-4,5-10$, and $11+)$. Similarly, public transit stops within 3 miles of each respondent were converted into quartiles $(0-110,111-464,465-879$, $880+$ stops).

Single-variable logistic models were used to select the 2- and 3-mile density measures for mammography facilities and public transportation respectively. As described earlier, ZIP Code, Census tract, HD, and SPA levels were examined to determine the most appropriate level of aggregation and each was tested in separate models; only SPA covariates were used in our final models.

All possible two-way interactions of the main effects were tested for significance at the $\alpha=0.0001$ level using a fixed effects logistic forward stepwise selection procedure [38]. This criterion is more stringent than the usual $\alpha=0.05$ level in order to adjust for multiple comparisons [39].

Our final analysis used a logistic generalized linear mixed effects model [40]. Additional variance components were included to account for overdispersion and spatial autocorrelation. Our final four models were chosen after comparing the AIC statistic [37] and goodness of fit, as determined by residual analysis and by the Hosmer-Lemeshow statistic [41]. A three-way interaction of Hispanic ethnicity, age, and usual source of care was added to improve model fit, as suggested by the residual analysis. Separate models were used to test the importance of the practice setting and social context covariates.

\section{Results}

Only the variables that met the theoretical and statistical criteria described earlier and that were selected for the logistic generalized linear mixed effects model are discussed, and only statistically significant results are reported. We present our results organized by the levels of analysis in our conceptual model. 
Table 2 Sample characteristics-CHIS 2001-Los Angeles County women aged 40-84 $(n=4,249)$

\begin{tabular}{|c|c|}
\hline \multicolumn{2}{|l|}{ Patient characteristics } \\
\hline Sociodemographics & Number $(\%)$ \\
\hline \multicolumn{2}{|l|}{ Race/ethnicity } \\
\hline Latina & $750(17.7)$ \\
\hline Black & $492(11.6)$ \\
\hline Asian/Pacific Islander & $517(12.2)$ \\
\hline Other & $162(3.8)$ \\
\hline White & $2,328(54.7)$ \\
\hline \multicolumn{2}{|l|}{ Age } \\
\hline $40-49$ & $1,553(36.5)$ \\
\hline $50-64$ & $1,560(36.7)$ \\
\hline $65-74$ & $639(15.0)$ \\
\hline $75-84$ & 497 (11.7) \\
\hline \multicolumn{2}{|l|}{ Education } \\
\hline$<$ High school & $681(16.0)$ \\
\hline High school graduate or GED & $1,017(23.9)$ \\
\hline Some college/technical school & $1,200(28.2)$ \\
\hline College or more & $1,351(31.8)$ \\
\hline \multicolumn{2}{|l|}{ Household income-midpoint categories } \\
\hline$\$ 3,000$ & $165(3.9)$ \\
\hline$\$ 8,000$ & $390(9.2)$ \\
\hline$\$ 13,000$ & $372(8.8)$ \\
\hline$\$ 18,000$ & $432(10.2)$ \\
\hline$\$ 25,000$ & $489(11.5)$ \\
\hline$\$ 35,000$ & $429(10.1)$ \\
\hline$\$ 45,000$ & $403(9.5)$ \\
\hline$\$ 55,000$ & $262(6.2)$ \\
\hline$\$ 65,000$ & $228(5.4)$ \\
\hline$\$ 75,000$ & $227(5.3)$ \\
\hline$\$ 85,000$ & $147(3.5)$ \\
\hline$\$ 95,000$ & $145(3.4)$ \\
\hline$\$ 118,000$ & $223(5.2)$ \\
\hline$\$ 168,000$ & $337(7.9)$ \\
\hline \multicolumn{2}{|l|}{ Marital status } \\
\hline Married or living with partner & $2,209(52.0)$ \\
\hline Divorced, separated, widowed, never married & $2,040(48.0)$ \\
\hline \multicolumn{2}{|l|}{ English proficiency } \\
\hline Not at all/not very well & $693(16.3)$ \\
\hline Very well/English only & $3,556(83.7)$ \\
\hline \multicolumn{2}{|l|}{ Health and health care access } \\
\hline \multicolumn{2}{|l|}{ History of breast cancer } \\
\hline No & $3,782(89.0)$ \\
\hline Yes & $467(11.0)$ \\
\hline \multicolumn{2}{|l|}{ Self-rated health } \\
\hline Poor/fair & $1,032(24.3)$ \\
\hline Good & $1,247(29.3)$ \\
\hline Very good/excellent & $1,970(46.4)$ \\
\hline
\end{tabular}

Table 2 continued

\begin{tabular}{|c|c|}
\hline \multicolumn{2}{|l|}{ Usual source of care } \\
\hline No & $317(7.5)$ \\
\hline Yes & $3,932(92.5)$ \\
\hline \multicolumn{2}{|l|}{ Insurance status } \\
\hline No & $612(14.4)$ \\
\hline Yes & $3,637(85.6)$ \\
\hline \multicolumn{2}{|l|}{ Social context/community level } \\
\hline \multicolumn{2}{|l|}{$\begin{array}{l}\text { Person-centered access (distancel } \\
\text { GIS variables) }\end{array}$} \\
\hline \multicolumn{2}{|l|}{$\begin{array}{l}\text { Nearest mammography facility } \\
\text { (Euclidean) }\end{array}$} \\
\hline $\begin{array}{l}\text { Quartile } 1 \text { (closest) } \\
\text { (03-0.53 miles) }\end{array}$ & $1,060(24.9)$ \\
\hline Quartile 2 (0.54-1.07 miles) & $1,062(25.0)$ \\
\hline Quartile 3 (1.08-1.82 miles) & $1,062(25.0)$ \\
\hline $\begin{array}{l}\text { Quartile } 4 \text { (reference) } \\
\quad(1.83-26.5 \text { miles })\end{array}$ & $1,065(25.1)$ \\
\hline $\begin{array}{l}\text { Number of FDA mammography } \\
\text { facilities within } 2 \text { miles of } \\
\text { respondent }\end{array}$ & $\begin{array}{l}\operatorname{Min}=0 / \max =57 \\
\quad \text { mean }=7.6(\mathrm{SD}=8.9)\end{array}$ \\
\hline $\begin{array}{l}\text { Number of public transit stops within } \\
3 \text { miles of respondent }\end{array}$ & $\begin{array}{l}\text { Min }=0 / \max =4,582 \\
\quad \text { mean }=644(\mathrm{SD}=789)\end{array}$ \\
\hline
\end{tabular}

Practice setting/individual level.

1. Patient characteristics

Description of sample (see Table 2)

Sociodemographics: Fifty-five percent of women aged 4084 in LA County reported white race/ethnicity, 18\% reported Latina, and $12 \%$ each reported black or Asian/ Pacific Islander. Roughly $1 / 3$ of women were $40-49,1 / 3$ were 50-64, and 1/3 were 65 and older. Forty percent reported either being a high school graduate or having less than a high school education; $28 \%$ some college or technical school; and $32 \%$ reported a college education or more. Fifty-four percent of our study sample reported an annual income less than the $\$ 35,000$ midpoint. About an even percentage were married or living with a partner vs. being single. Sixteen percent were not English proficient.

Health status and health care access variables: Eleven percent reported a history of breast cancer. Forty-six percent reported being in very good or excellent health. Eight percent reported no usual source of care and fourteen percent no insurance coverage.

Person-centered access: Within a 2-mile buffer around each respondent's home address, there was a mean of eight mammography facilities. There was a mean of 644 transit stops within a 3-mile buffer around each respondent's home address. 
Table 3 Odds ratios for women aged 40-84 having received a mammogram in past 2 years in Los Angeles County 2001

\begin{tabular}{|c|c|c|c|c|c|c|c|c|}
\hline \multirow[t]{3}{*}{$n=4,249$} & \multicolumn{2}{|c|}{ Model 1} & \multicolumn{2}{|c|}{ Model 2} & \multicolumn{2}{|c|}{ Model 3} & \multicolumn{2}{|c|}{ Model 4} \\
\hline & $\begin{array}{l}\text { Odds } \\
\text { ratios }\end{array}$ & $\begin{array}{l}95.0 \% \text { CI for } \\
\text { EXP(B) }\end{array}$ & $\begin{array}{l}\text { Odds } \\
\text { ratios }\end{array}$ & $\begin{array}{l}95.0 \% \mathrm{CI} \\
\text { for } \operatorname{EXP}(\mathrm{B})\end{array}$ & $\begin{array}{l}\text { Odds } \\
\text { ratios }\end{array}$ & $\begin{array}{l}95.0 \% \mathrm{CI} \\
\text { for } \operatorname{EXP}(\mathrm{B})\end{array}$ & $\begin{array}{l}\text { Odds } \\
\text { ratios }\end{array}$ & $\begin{array}{l}95.0 \% \mathrm{CI} \\
\text { for } \operatorname{EXP}(\mathrm{B})\end{array}$ \\
\hline & & Lower Upper & & Lower Upper & & Lower Upper & & Lower Upper \\
\hline
\end{tabular}

A. Practice setting

1. Patient characteristics

Sociodemographics

Race/ethnicity

Latina

$\begin{array}{llllllllllll}1.199 & 0.921 & 1.560 & 1.209 & 0.928 & 1.574 & 1.219 & 0.933 & 1.593 & 1.206 & 0.924 & 1.575\end{array}$

Black

$\begin{array}{lllllllllllll}1.253 & 0.969 & 1.619 & 1.273 & 0.982 & 1.652 & 1.320 & 0.984 & 1.770 & 1.270 & 0.968 & 1.667\end{array}$

Asian/Pacific Islander

$\begin{array}{lllllllllllll}0.605 & 0.476 & 0.770 & 0.640 & 0.500 & 0.817 & 0.636 & 0.496 & 0.815 & 0.635 & 0.496 & 0.813\end{array}$

Other

White

0.6740 .469

0.6910 .478

0.99

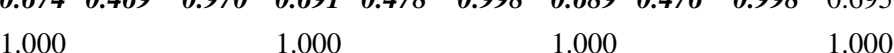

Age

40-49

$\begin{array}{lll}0.509 & 0.412 & 0.630\end{array}$

50-64

65-84 (reference)

$\begin{array}{lll}1.102 & 0.889 & 1.365\end{array}$

1.000

Education

$<$ High school

High school graduate or GED

Some college/technical school

College or more (reference)

Household income-midpoint categories

$\$ 3,000$

$\$ 8,000$

$\$ 13,000$

$\$ 18,000$

$\$ 25,000$

$\$ 35,000$

$\$ 45,000$

$\$ 55,000$

$\$ 65,000$

$\$ 75,000$

$\$ 85,000$

$\$ 95,000$

$\$ 118,000$

$\$ 168,000$ (reference)

Marital status

Divorced, separated, widowed, never married (reference)

Married or living with partner

$\begin{array}{llllllllllll}0.889 & 0.660 & 1.198 & 0.879 & 0.651 & 1.188 & 0.892 & 0.658 & 1.208 & 0.887 & 0.656 & 1.199 \\ 0.940 & 0.757 & 1.168 & 0.916 & 0.736 & 1.140 & 0.926 & 0.742 & 1.156 & 0.930 & 0.746 & 1.159 \\ 1.125 & 0.918 & 1.380 & 1.133 & 0.921 & 1.393 & 1.143 & 0.928 & 1.408 & 1.142 & 0.928 & 1.406 \\ 1.000 & & & 1.000 & & & 1.000 & & & 1.000 & & \end{array}$

$\begin{array}{llllllllllll}0.426 & 0.264 & 0.687 & 0.417 & 0.257 & 0.677 & 0.417 & 0.256 & 0.678 & 0.408 & 0.250 & 0.664\end{array}$

$\begin{array}{lllllllllllll}0.595 & 0.391 & 0.906 & 0.625 & 0.408 & 0.958 & 0.621 & 0.404 & 0.953 & 0.610 & 0.397 & 0.937\end{array}$

$\begin{array}{lllllllllllll}0.749 & 0.497 & 1.131 & 0.745 & 0.491 & 1.130 & 0.741 & 0.488 & 1.127 & 0.727 & 0.478 & 1.105\end{array}$

$\begin{array}{lllllllllllll}0.633 & 0.429 & 0.934 & 0.591 & 0.398 & 0.877 & 0.592 & 0.398 & 0.879 & 0.576 & 0.388 & 0.857\end{array}$

$\begin{array}{llllllllllll}0.729 & 0.502 & 1.060 & 0.697 & 0.477 & 1.018 & 0.701 & 0.479 & 1.026 & 0.695 & 0.475 & 1.017\end{array}$

$\begin{array}{llllllllllll}0.807 & 0.554 & 1.178 & 0.789 & 0.539 & 1.155 & 0.792 & 0.540 & 1.161 & 0.783 & 0.534 & 1.148\end{array}$

$\begin{array}{llllllllllll}0.871 & 0.598 & 1.267 & 0.851 & 0.583 & 1.243 & 0.858 & 0.587 & 1.255 & 0.849 & 0.581 & 1.240\end{array}$

$\begin{array}{llllllllllll}0.979 & 0.642 & 1.493 & 0.956 & 0.624 & 1.463 & 0.959 & 0.626 & 1.470 & 0.963 & 0.629 & 1.477\end{array}$

$\begin{array}{llllllllllll}0.921 & 0.599 & 1.415 & 0.916 & 0.593 & 1.413 & 0.919 & 0.595 & 1.420 & 0.917 & 0.593 & 1.417\end{array}$

$\begin{array}{lllllllllllll}1.136 & 0.731 & 1.764 & 1.139 & 0.730 & 1.776 & 1.143 & 0.732 & 1.785 & 1.144 & 0.733 & 1.786\end{array}$

$\begin{array}{lllllllllllll}0.929 & 0.566 & 1.524 & 0.930 & 0.564 & 1.532 & 0.940 & 0.569 & 1.551 & 0.961 & 0.582 & 1.587\end{array}$

$\begin{array}{lllllllllllll}0.930 & 0.567 & 1.525 & 0.895 & 0.545 & 1.470 & 0.902 & 0.549 & 1.481 & 0.894 & 0.544 & 1.469\end{array}$

$\begin{array}{lllllllllllll}1.255 & 0.800 & 1.971 & 1.303 & 0.826 & 2.056 & 1.309 & 0.829 & 2.068 & 1.312 & 0.830 & 2.072\end{array}$

$\begin{array}{llll}1.000 & 1.000 & 1.000 & 1.000\end{array}$

$\begin{array}{lll}1.000 & 1.000 & 1.000\end{array}$

$\begin{array}{llllllllllll}1.150 & 0.972 & 1.360 & 1.156 & 0.975 & 1.370 & 1.155 & 0.974 & 1.370 & 1.172 & 0.988 & 1.390\end{array}$

English proficiency

Not at all/not very well (reference)

1.000

Very well/english only

$\begin{array}{lll}0.768 & 0.582 & 1.013\end{array}$

Health and health care access

History of breast cancer

Yes

$\begin{array}{llllllllllll}1.607 & 1.227 & 2.106 & 1.629 & 1.238 & 2.144 & 1.627 & 1.236 & 2.142 & 1.616 & 1.228 & 2.128\end{array}$ 
Table 3 continued

\begin{tabular}{|c|c|c|c|c|c|c|c|c|c|c|c|c|}
\hline \multirow[t]{3}{*}{$n=4,249$} & \multicolumn{3}{|c|}{ Model 1} & \multicolumn{3}{|c|}{ Model 2} & \multicolumn{3}{|c|}{ Model 3} & \multicolumn{3}{|c|}{ Model 4} \\
\hline & \multirow[t]{2}{*}{$\begin{array}{l}\text { Odds } \\
\text { ratios }\end{array}$} & \multicolumn{2}{|c|}{$\begin{array}{l}95.0 \% \text { CI for } \\
\operatorname{EXP(B)}\end{array}$} & \multirow[t]{2}{*}{$\begin{array}{l}\text { Odds } \\
\text { ratios }\end{array}$} & \multicolumn{2}{|c|}{$\begin{array}{l}95.0 \% \mathrm{CI} \\
\text { for } \operatorname{EXP}(\mathrm{B})\end{array}$} & \multirow[t]{2}{*}{$\begin{array}{l}\text { Odds } \\
\text { ratios }\end{array}$} & \multicolumn{2}{|c|}{$\begin{array}{l}95.0 \% \mathrm{CI} \\
\text { for } \operatorname{EXP}(\mathrm{B})\end{array}$} & \multirow[t]{2}{*}{$\begin{array}{l}\text { Odds } \\
\text { ratios }\end{array}$} & \multicolumn{2}{|c|}{$\begin{array}{l}95.0 \% \mathrm{CI} \\
\text { for } \operatorname{EXP}(\mathrm{B})\end{array}$} \\
\hline & & Lower & Upper & & Lower & Upper & & Lower & Upper & & Lower & Upper \\
\hline \multicolumn{13}{|l|}{ Self-rated health } \\
\hline Poor/fair & 0.996 & 0.807 & 1.228 & 0.980 & 0.792 & 1.213 & 0.980 & 0.791 & 1.214 & 0.981 & 0.792 & 1.215 \\
\hline Good & 0.952 & 0.795 & 1.141 & 0.933 & 0.777 & 1.120 & 0.935 & 0.779 & 1.123 & 0.945 & 0.786 & 1.135 \\
\hline Very good/excellent (reference) & 1.000 & & & 1.000 & & & 1.000 & & & 1.000 & & \\
\hline \multicolumn{13}{|l|}{ Usual source of care } \\
\hline Yes & 3.173 & 2.443 & 4.121 & & & & & & & & & \\
\hline \multicolumn{13}{|l|}{ Insurance status } \\
\hline Yes & 1.531 & 1.223 & 1.917 & 1.678 & 1.335 & 2.108 & 1.686 & 1.341 & 2.119 & 1.686 & 1.340 & 2.120 \\
\hline \multicolumn{13}{|l|}{ Interactions } \\
\hline \multicolumn{13}{|l|}{ English proficiency $=$ very well/English only } \\
\hline \multicolumn{13}{|l|}{ Usual source of care $=$ yes } \\
\hline Age $40-49$ & & & & 0.444 & 0.362 & 0.545 & 0.443 & 0.361 & 0.544 & 0.443 & 0.361 & 0.544 \\
\hline Age 50-64 (reference) & & & & 1.000 & & & 1.000 & & & 1.000 & & \\
\hline Age $65-84$ & & & & 1.119 & 0.878 & 1.425 & 1.127 & 0.884 & 1.436 & 1.126 & 0.883 & 1.436 \\
\hline Usual source of care $=$ no & & & & & & & & & & & & \\
\hline Age $40-49$ & & & & 0.246 & 0.158 & 0.381 & 0.244 & 0.157 & 0.379 & 0.241 & 0.155 & 0.375 \\
\hline Age $50-64$ & & & & 0.148 & 0.086 & 0.256 & 0.148 & 0.086 & 0.257 & 0.150 & 0.087 & 0.260 \\
\hline Age $65-84$ & & & & 0.065 & 0.025 & 0.169 & 0.066 & 0.025 & 0.173 & 0.061 & 0.023 & 0.159 \\
\hline English proficiency $=$ not very well/not a & & & & & & & & & & & & \\
\hline Usual source of care $=$ yes & & & & & & & & & & & & \\
\hline Age $40-49$ & & & & 0.640 & 0.438 & 0.937 & 0.641 & 0.438 & 0.940 & 0.637 & 0.434 & 0.933 \\
\hline Age $50-64$ & & & & 1.895 & 1.182 & 3.040 & 1.894 & 1.180 & 3.041 & 1.849 & 1.151 & 2.971 \\
\hline Age $65-84$ & & & & 0.554 & 0.350 & 0.877 & 0.549 & 0.346 & 0.871 & 0.547 & 0.345 & 0.869 \\
\hline Usual source of care $=$ no & & & & & & & & & & & & \\
\hline Age $40-49$ & & & & 0.228 & 0.128 & 0.407 & 0.228 & 0.128 & 0.408 & 0.225 & 0.126 & 0.403 \\
\hline Age $50-64$ & & & & 0.784 & 0.417 & 1.472 & 0.777 & 0.413 & 1.462 & 0.751 & 0.398 & 1.417 \\
\hline Age $65-84$ & & & & 1.164 & 0.101 & 13.474 & 1.168 & 0.101 & 13.575 & 1.127 & 0.097 & 13.116 \\
\hline 2. Clinician/team characteristics & & & & & & & & & & & & \\
\hline$\%$ Board certified & & & & & & & 1.012 & 0.980 & 1.045 & & & \\
\hline$\%$ Primary care provider & & & & & & & 0.999 & 0.992 & 1.006 & & & \\
\hline$\%$ Solo practitioner & & & & & & & 1.005 & 0.984 & 1.026 & & & \\
\hline \# Years since graduation & & & & & & & 1.005 & 0.990 & 1.021 & & & \\
\hline 3. Encounters/interactions & & & & & & & & & & & & \\
\hline Index of doctor/patient relationships & & & & & & & 1.152 & 0.812 & 1.634 & & & \\
\hline Index of ability to obtain quality services & & & & & & & 0.988 & 0.729 & 1.339 & & & \\
\hline B. Plan level (medical group) & & & & & & & & & & & & \\
\hline$\%$ Accept no MediCal & & & & & & & 0.985 & 0.959 & 1.011 & & & \\
\hline C. Social context/community level & & & & & & & & & & & & \\
\hline Person-centered access & & & & & & & & & & & & \\
\hline (Distance/GIS variables) & & & & & & & & & & & & \\
\hline $\begin{array}{l}\text { Number of FDA mammography facilities } \\
2 \text { miles of respondent (quartiles) }\end{array}$ & & & & & & & & & & & & \\
\hline Quartile $1(0-1)$ & & & & & & & & & & 0.762 & 0.600 & 0.967 \\
\hline Quartile $2(2-4)$ & & & & & & & & & & 0.976 & 0.763 & 1.248 \\
\hline Quartile $3(5-10)$ & & & & & & & & & & 0.805 & 0.650 & 0.997 \\
\hline Quartile $4(11+)$ (reference) & & & & & & & & & & 1.000 & & \\
\hline
\end{tabular}


Table 3 continued

\begin{tabular}{|c|c|c|c|c|c|c|c|c|c|c|}
\hline \multirow[t]{3}{*}{$n=4,249$} & \multicolumn{2}{|l|}{ Model 1} & \multicolumn{3}{|c|}{ Model 2} & \multicolumn{2}{|c|}{ Model 3} & \multicolumn{3}{|c|}{ Model 4} \\
\hline & \multirow[t]{2}{*}{$\begin{array}{l}\text { Odds } \\
\text { ratios }\end{array}$} & $\begin{array}{l}95.0 \% \text { CI for } \\
\operatorname{EXP}(B)\end{array}$ & \multirow[t]{2}{*}{$\begin{array}{l}\text { Odds } \\
\text { ratios }\end{array}$} & \multicolumn{2}{|c|}{$\begin{array}{l}95.0 \% \mathrm{CI} \\
\text { for } \operatorname{EXP}(\mathrm{B})\end{array}$} & \multirow[t]{2}{*}{$\begin{array}{l}\text { Odds } \\
\text { ratios }\end{array}$} & $\begin{array}{l}95.0 \% \mathrm{CI} \\
\text { for } \operatorname{EXP}(\mathrm{B})\end{array}$ & \multirow[t]{2}{*}{$\begin{array}{l}\text { Odds } \\
\text { ratios }\end{array}$} & \multicolumn{2}{|c|}{$\begin{array}{l}95.0 \% \mathrm{CI} \\
\text { for } \operatorname{EXP}(\mathrm{B})\end{array}$} \\
\hline & & Lower Upper & & Lower & Upper & & Lower Upper & & Lower & Upper \\
\hline \multicolumn{11}{|c|}{$\begin{array}{l}\text { Number of public transit stops within } 3 \text { miles of } \\
\text { respondent (quartiles) }\end{array}$} \\
\hline Quartile $1(0-110)$ & & & & & & & & 1.050 & 0.821 & 1.342 \\
\hline Quartile 2 (111-464) & & & & & & & & 0.876 & 0.687 & 1.116 \\
\hline Quartile 3 (465-879) & & & & & & & & 0.953 & 0.762 & 1.191 \\
\hline \multirow[t]{2}{*}{ Quartile $4(880+)$ (reference) } & & & & & & & & 1.000 & & \\
\hline & AIC & & & & & & & & & \\
\hline Model 1 & $4,429.5$ & & & & & & & & & \\
\hline Model 2 & $4,387.91$ & & & & & & & & & \\
\hline Model 3 & $4,397.6$ & & & & & & & & & \\
\hline Model 4 & $4,386.66$ & & & & & & & & & \\
\hline
\end{tabular}

Bold italic values represent $p<0.05$

Table 3 presents odds ratios for the sociodemographic, practice setting, and community variables. Four models are presented. The first tests individual-level variables. The second model also includes individual-level interactions. The third model adds variables that describe clinician/team characteristics, encounters between patients and providers and plan-level characteristics. The fourth model adds social context and community level variables. These include measures of density of mammography facilities and bus stops. None of the clinician/team characteristics proved significant so they were left out of the fourth model.

Individual characteristics examined in Model 1 were based on findings from previous literature on mammography [42-46]. In Model 1, women who reported their race as Asian/Pacific Islander were less likely to report having a mammogram within the previous 2 years $(\mathrm{OR}=0.61)$ compared with other women. The small proportion of women grouped into "other" race also was less likely to be on-schedule for breast cancer screening $(\mathrm{OR}=0.67)$.

Women 40-49 years of age were less likely to report having a mammogram within the past 2 years $(\mathrm{OR}=0.51)$ compared with women 65-84 years of age. Women with low income were less likely to have had a recent mammogram $(\$ 3,000$ midpoint $\mathrm{OR}=0.43 ; \$ 8,000$ midpoint $\mathrm{OR}=0.60$; and $\$ 18,000$ midpoint $\mathrm{OR}=0.63$ ) than the most affluent respondents in our CHIS sample.

Women with a previous breast cancer diagnosis $(\mathrm{OR}=1.61)$, a usual source of health care $(\mathrm{OR}=3.17)$, or insurance coverage in the past 12 months $(\mathrm{OR}=1.53)$ were significantly more likely than others to report a recent mammogram.

Significant interactions between age, English proficiency, and having a usual source of health care were found for Models 2-4. Of the odds ratios shown in Table 3 for these combinations of individual characteristics, the pattern among women ages 50-64 is unusual. Women in this age group who had a usual source of health care and had limited English proficiency (predominantly Latina) were significantly more likely $(\mathrm{OR}=1.85)$ to have a recent mammogram than English-proficient women of the same age who had a usual source of care.

2. Clinician/team characteristics

3. Encounters/interactions between doctors and patients

None of the variables that represent clinician/team characteristics and encounters/interactions within the practice setting were significant in our results (Model 3 in Table 3).

Plan or medical group level

Additionally, the plan or medical group level was not significant in our results (Model 3 in Table 3 ).

Social and economic context/community level

Model 4, which includes person-centered access variables of mammography and transportation density, was the best model as indicated by the smaller AIC statistic. Model 4 was slightly better than Model 2 which was identical except for the person-centered variables. Model 4 showed increasing likelihood of a recent mammogram as the number of mammography facilities within 2 miles of the respondent's home increased. Women with the fewest number of nearby facilities (none or 1) had the lowest odds ratio $(\mathrm{OR}=0.76)$ compared with those with 11 or more facilities nearby. Women with 2-4 and 5-10 facilities nearby had intermediate odds ratios $(\mathrm{OR}=0.98$ and 0.81 , respectively). The interrupted gradient of density of facilities to mammography use may be due to SES differences 
and unmeasured factors such as proximity of a mammography facility to a women's workplace or other locations she frequents. It may also mean that there is little difference between having 2-10 facilities nearby. Density of public transportation stops within 3 miles of a respondent's residence was not associated with mammography use.

\section{Discussion}

Our study examined area-level and individual-level effects on mammography use for women in Los Angeles County. Gumpertz et al. had found that a longer distance from the population center of the census tract to the nearest mammography facility predicted more advanced disease for Latina and White women after controlling for other important factors [14]. This led us to hypothesize that a lack of nearby mammography facilities would lead to less mammography use, which in turn would lead to a laterstage diagnosis of breast cancer. Consistent with this hypothesis, we found an increased use of mammography when many facilities were nearby. At the same time, our study confirmed the significance of individual characteristics found to be important predictors of mammography use in other studies [42-46].

Our most striking and unexpected findings were revealed by the interactions. Not surprisingly, women aged 40-49 were less likely to have had a mammogram than women aged 50-64 - an age group for which mammography is recommended by evidence-based guidelines in the United States [47]. For women under 65 not covered by Medicare, lack of usual source of care is usually strongly associated with lower mammography rates. However, among the 50-64 years old group who had a usual source of health care in Los Angeles, we found that women with limited or no English proficiency were more likely than English-proficient women to obtain a mammogram. These unexpected findings were consistent across all the models.

To help explain this finding, we examined data on 127,000 women screened in Los Angeles during 19992001 by their race-ethnicity from the Cancer Detection Section (CDS) of the California Department of Health Services. Comparing the percent of eligible women who actually used the program to those eligible, we found proportional underrepresentation among whites (18\% eligible and $7 \%$ users of program) and blacks (9\% eligible and $3 \%$ users); and overrepresentation among Latinas (64\% eligible and $70 \%$ users) and Asian/Pacific Islanders (API) (9 percent eligible and 11 percent users). Further investigation showed that the Every Women Counts Program (EWC), directed by a community-based organization in Los Angeles County, had conducted outreach between 1999 and 2001 that targeted Spanish-speaking Latinas. The
EWC program, co-funded by the CDC BCCEDP and California state, provides subsidized breast and cervical cancer screening services to low income underinsured residents. The program's education and outreach to Spanish-speaking Latinas comprised a number of different strategies including an "over 50 task force" and a "grandmother's campaign" with community participation on task forces and committees (Brian Montano, personal communication, 1 May 2008). The program sponsored a Mother's Day campaign in which providers were given reminder cards to use with their patients. Providers were encouraged to emphasize the importance of screening and re-screening to community members. The program also helped providers participating in the EWC Program develop manual and computerized tracking systems to notify women that they were due for screening (Patricia Smith Francis, personal communication, 29 August 2008). This reliance on health care providers to encourage women to be screened, with a particular emphasis on Latinas over age 50, is probably why English fluency, age, and usual source of care significantly interacted to predict mammography use in our models.

Our results suggest that this campaign succeeded in reaching communities in need. The communities targeted in the outreach campaigns were the same ones that Gumpertz reported were disproportionately impacted by late stage breast cancer. Previous literature has shown that organized communities and social networks can effectively promote use of services, including cancer screening [48, 49]. Our findings for LA County in the context of the community-based outreach program to promote mammography among Latinas confirmed this.

Our analysis confirmed low use of mammography by Asian women, women 40-49 and lower-income women in Los Angeles [50, 51]. We confirmed greater use by women in the 50-64 and 65-84 age groups, those with a personal history of breast cancer, and those with a usual source of care and health insurance coverage. Previous findings have shown these individual variables are correlated with recent mammography use both for the nation and for Los Angeles [52, 53].

Our study is the first to show an association between use of mammography and density of mammography facilities within 2 miles of a woman's residence. We also examined an alternative measure, distance of mammography facilities in relation to a respondent's residence. This is the measure used in the Gumpertz et al. article; however, odds ratios using this measure were small and not statistically significant (data not shown) so we used the mammography density measure instead.

Our study benefitted from having an address for each woman and for each mammography facility, from which more precise locations and associations could be measured [54]. Most previous studies, including that of Gumpertz 
et al. have assigned respondents to aggregated geographic units, such as Census tracts, counties, or zip codes. Krieger et al. have shown that it is important to compare socioeconomic status using small geographic units such as tracts in order to identify health inequalities [55].

The above finding suggests that a woman's proximity to the closest facility may not be as important as living in an area with a greater density of facilities. As indicated in the methods section, the density variable potentially captures more aspects of access than the proximity variable and may explain why only the density variable is associated with mammography use. Another possible explanation is that using the exact location of residence as we did in our study is a more accurate measure compared with using a Census tract centroid in the calculation of proximity, the address surrogate used in the Gumpertz study. Our finding that density of mammography facilities is an important determinant of mammography use suggests that examining the supply of mammography facilities, including capacity, location and staffing, needs additional study.

\section{Limitations}

The geocoded location of each woman was based on her reported nearest street intersection, not her exact address. The geocoding process has been shown to introduce some positional inaccuracies, but resulting locations are generally within $100 \mathrm{~m}$ of the true location [56]. Because most of LA County is very urban, we expect that most of the geocoded locations are very close to the actual residence. However, location of workplace was not available from the CHIS survey. It is likely that some women who work would find it convenient to have their mammograms at facilities close to their workplace, especially if there were few facilities available near their home.

Transit data throughout LA County were incomplete because data on rail lines run by Municipal Operators and Foothill Transit in LA County were not available. However, the Metro Transit Authority (MTA) estimates that these rail lines would add only $15 \%$ more rail stops to the present analysis (Dr. Jesse Simmon, personal communication, 24 January 2005).

The distribution of data was not adequate at all levels of geography. This limited our ability to test some variables. Though we do not know exactly how this limitation affected our analysis, it is likely that the sample of physician practice characteristics was too small to provide adequate power to detect differences in Los Angeles. In addition, CHIS data are self-reported and previous analyses have shown that mammography may be over-reported in surveys, particularly among racial/ethnic groups $[57,58]$.

\section{Conclusions}

Women with limited English proficiency were especially likely to report recent mammography in Los Angeles. This unexpected finding suggests that the intensive Spanishlanguage outreach program conducted by the EWC Program in low-income Latina communities in Los Angeles was effective. Our spatial analysis of mammography use in Los Angeles was inspired by Gumpertz et al. who found that Latinas in Los Angeles County were diagnosed with later stage breast cancer than other women (1992-1996) and that distance from the population center of the Census tract to the nearest mammography facility was a significant predictor of advanced disease. Our study highlights the success of the targeted community-based outreach conducted between 1999 and 2001 which addressed the same populations that Gumpertz et al. identified as needing intervention. It would be useful to conduct another study of late-stage diagnosis in Los Angeles County to ascertain whether increased rates of mammography also led to less late-stage diagnosis among Latinas in the neighborhoods where they are concentrated in Los Angeles.

Open Access This article is distributed under the terms of the Creative Commons Attribution Noncommercial License which permits any noncommercial use, distribution, and reproduction in any medium, provided the original author(s) and source are credited.

\section{References}

1. Rauscher GH, Johnson TP, Cho YI, Walk JA (2008) Accuracy of self-reported cancer-screening histories: a meta-analysis. Cancer Epidemiol Biomarkers Prev 17:748-757. doi:10.1158/10559965.EPI-07-2629

2. Breen N, Yabroff KR, Meissner HI (2007) What proportion of breast cancers are detected by mammography in the United States? Cancer Detect Prev 31:220-224. doi:10.1016/j.cdp.2007. 04.006

3. Jones AR, Caplan LS, Davis MK (2003) Racial/ethnic differences in the self-reported use of screening mammography. J Community Health 28:303-316. doi:10.1023/A:1025451412007

4. Ryerson AB, Miller JW, Eheman CR, Leadbetter S, White MC (2008) Recent trends in US mammography use from 2000-2006: a population-based analysis. Prev Med 47(5):477-482

5. Yabroff KR, Freedman A, Brown ML, Ballard-Barbash R, McNeel T, Taplin S (2007) Trends in abnormal cancer screening results in the United States of America. J Med Screen 14:67-72. doi:10.1258/096914107781261909

6. Yabroff KR, Breen N, Vernon SW, Meissner HI, Freedman AN, Ballard-Barbash R (2004) What factors are associated with diagnostic follow-up after abnormal mammograms? Findings from a US National Survey. Cancer Epidemiol Biomarkers Prev 13:723-732

7. Vogel VG (2008) Epidemiology, genetics, and risk evaluation of postmenopausal women at risk of breast cancer. Menopause 15:782-789. doi:10.1097/gme.0b013e3181788d88 
8. Graves KD, Huerta E, Cullen J et al. (2008) Perceived risk of breast cancer among Latinas attending community clinics: risk comprehension and relationship with mammography adherence. Cancer Causes Control 19(10):1373-1382

9. Sauaia A, Min SJ, Lack D et al (2007) Church-based breast cancer screening education: impact of two approaches on Latinas enrolled in public and private health insurance plans. Prev Chronic Dis 4:A99

10. Kudadjie-Gyamfi E, Magai C (2008) The influence of coping styles on mammography screening in a multiethnic sample. Cult Divers Ethnic Minor Psychol 14:183-192. doi:10.1037/10999809.14.3.183

11. Gross CP, Filardo G, Singh HS, Freedman AN, Farrell MH (2006) The relation between projected breast cancer risk, perceived cancer risk, and mammography use. Results from the National Health Interview Survey. J Gen Intern Med 21:158-164

12. Sabatino SA, Habarta N, Baron RC et al (2008) Interventions to increase recommendation and delivery of screening for breast, cervical, and colorectal cancers by healthcare providers systematic reviews of provider assessment and feedback and provider incentives. Am J Prev Med 35:S67-S74. doi:10.1016/j.amepre.2008. 04.008

13. Robinson JM, Shavers V (2008) The role of health insurance coverage in cancer screening utilization. J Health Care Poor Underserved 19:842-856. doi:10.1353/hpu.0.0048

14. Gumpertz ML, Pickle LW, Miller BA, Bell BS (2006) Geographic patterns of advanced breast cancer in Los Angeles: associations with biological and sociodemographic factors (United States). Cancer Causes Control 17:325-339. doi:10.1007/s10552-005-0513-1

15. O'Campo P, Xue X, Wang MC, Caughy M (1997) Neighborhood risk factors for low birthweight in Baltimore: a multilevel analysis. Am J Public Health 87:1113-1118. doi:10.2105/AJPH.87.7. 1113

16. Diez-Roux AV (2000) Multilevel analysis in public health research. Annu Rev Public Health 21:171-192. doi:10.1146/annurev.publ health.21.1.171

17. Barry J, Breen N (2005) The importance of place of residence in predicting late-stage diagnosis of breast or cervical cancer. Health Place 11:15-29. doi:10.1016/j.healthplace.2003.12.002

18. Bernard P, Charafeddine R, Frohlich KL, Daniel M, Kestens Y, Potvin L (2007) Health inequalities and place: a theoretical conception of neighbourhood. Soc Sci Med 65:1839-1852. doi: 10.1016/j.socscimed.2007.05.037

19. Cummins S, Curtis S, Diez-Roux AV, Macintyre S (2007) Understanding and representing 'place' in health research: a relational approach. Soc Sci Med 65:1825-1838. doi:10.1016/j.soc scimed.2007.05.036

20. Han D, Rogerson PA, Bonner MR et al (2005) Assessing spatiotemporal variability of risk surfaces using residential history data in a case control study of breast cancer. Int J Health Geogr 4:9. doi:10.1186/1476-072X-4-9

21. Bentley R, Kavanagh AM, Subramanian SV, Turrell G (2008) Area disadvantage, individual socio-economic position, and premature cancer mortality in Australia 1998 to 2000: a multilevel analysis. Cancer Causes Control 19:183-193. doi:10.1007/ s10552-007-9084-7

22. Sanderson M, Coker AL, Perez A, Du XL, Peltz G, Fadden MK (2006) A multilevel analysis of socioeconomic status and prostate cancer risk. Ann Epidemiol 16:901-907. doi:10.1016/j.annepidem. 2006.02.006

23. Waitzman NJ, Smith KR (1998) Separate but lethal: the effects of economic segregation on mortality in metropolitan America. Milbank Q 76:341-373. doi:10.1111/1468-0009.00095

24. Glass TA, McAtee MJ (2006) Behavioral science at the crossroads in public health: extending horizons, envisioning the future.
Soc Sci Med 62:1650-1671. doi:10.1016/j.socscimed.2005.08. 044

25. Zapka JG, Taplin SH, Solberg LI, Manos MM (2003) A framework for improving the quality of cancer care: the case of breast and cervical cancer screening. Cancer Epidemiol Biomarkers Prev 12: 4-13

26. Grady KE, Lemkau JP, McVay JM, Reisine ST (1992) The importance of physician encouragement in breast cancer screening of older women. Prev Med 21:766-780. doi:10.1016/00917435(92)90083-T

27. Lane DS, Zapka J, Breen N, Messina CR, Fotheringham DJ (2000) A systems model of clinical preventive care: the case of breast cancer screening among older women. Prev Med 31:481493. doi:10.1006/pmed.2000.0747

28. Lurie N, Slater J, McGovern P, Ekstrum J, Quam L, Margolis K (1993) Preventive care for women. Does the sex of the physician matter? N Engl J Med 329:478-482. doi:10.1056/NEJM19930 8123290707

29. Roetzheim RG, Fox SA, Leake B (1995) Physician-reported determinants of screening mammography in older women: the impact of physician and practice characteristics. J Am Geriatr Soc 43:1398-1402

30. Ward MM, Vaughn TE, Uden-Holman T, Doebbeling BN, Clarke WR, Woolson RF (2002) Physician knowledge, attitudes and practices regarding a widely implemented guideline. J Eval Clin Pract 8:155-162. doi:10.1046/j.1365-2753.2002.00337.x

31. Mandelblatt J, Kanetsky PA (1995) Effectiveness of interventions to enhance physician screening for breast cancer. J Fam Pract 40: $162-171$

32. Rimer BK (1998) Interventions to enhance cancer screening. Cancer 83:1770-1774. doi:10.1002/(SICI)1097-0142(19981015)83:8+<17 70::AID-CNCR20>3.0.CO;2-7

33. US Food and Drug Administration (2005) Mammography program within the Center for Devices and Radiological Health. Available at: http://www.accessdata.fda.gov/scripts/cdrh/cfdocs/ cfMQSA/mqsa.cfm. Accessed June 2006

34. Los Angeles County Metro Transit Authority (2005) Data accessed through program correspondence 2/15/2005

35. US Census Bureau (2000) Census 2000 summary file 3 (SF 3) sample data. Available at http://factfinder.census.gov/servlet/Data setMainPageServlet?_ds_name=DEC_2000_SF3_U\&_program= DEC\&_lang=en. Accessed June 2006

36. Meersman SC (2005) Objective neighborhood properties and perceptions of neighborhood problems: using a geographic information system (GIS) in neighborhood effects and aging research. Ageing Int 30:63-87. doi:10.1007/BF02681007

37. Akaike H (1973) Information theory and an extension of the maximum likelihood principle. In: Petrov BN, Csaki F (eds) Proceedings of the 2 nd international symposium on information theory. Akademiai Kiado, Budapest, pp 267-281

38. SAS Institute Inc. (1999) SAS/STAT user's guide, version 8. SAS Institute, Inc., Cary, NC

39. Christensen LR (1973) Simultaneous statistical inference in the normal multiple linear regression model. J Am Stat Assoc 68: 457-461. doi:10.2307/2284098

40. SAS Institute Inc (2006) SAS/STAT software: documentation for the 9.1 release of the GLIMMIX Procedure. Available at http://www.sas.com/statistics/doc.html. Accessed 27 March 2008

41. Hosmer DW, Lemeshow S (1980) A goodness-of-fit test for the multiple logistic regression model. Commun Stat A10:10431069. doi:10.1080/03610928008827941

42. Calvocoressi L, Sun A, Kasl SV, Claus EB, Jones BA (2008) Mammography screening of women in their $40 \mathrm{~s}$ : impact of changes in screening guidelines. Cancer 112:473-480. doi:10.1002/cncr. 23210 
43. Keating NL, Landrum MB, Guadagnoli E, Winer EP, Ayanian JZ (2006) Factors related to underuse of surveillance mammography among breast cancer survivors. J Clin Oncol 24:85-94. doi: 10.1200/JCO.2005.02.4174

44. Meissner HI, Breen N, Taubman ML, Vernon SW, Graubard BI (2007) Which women aren't getting mammograms and why? (United States). Cancer Causes Control 18:61-70. doi:10.1007/ s10552-006-0078-7

45. Coughlin SS, Leadbetter S, Richards T, Sabatino SA (2008) Contextual analysis of breast and cervical cancer screening and factors associated with health care access among United States women, 2002. Soc Sci Med 66:260-275. doi:10.1016/j.socscimed.2007. 09.009

46. Rakowski W, Meissner H, Vernon SW, Breen N, Rimer B, Clark MA (2006) Correlates of repeat and recent mammography for women ages 45 to 75 in the 2002 to 2003 Health Information National Trends Survey (HINTS 2003). Cancer Epidemiol Biomarkers Prev 15:2093-2101. doi:10.1158/1055-9965.EPI-06-0301

47. US Preventive Services Task Force (2003) Screening for breast cancer, topic page. Agency for Healthcare Research and Quality. Available at http://www.ahrq.gov/clinic/uspstf/uspsbrca.htm. Accessed 30 April 2008

48. Jandorf L, Fatone A, Borker PV et al (2006) Creating alliances to improve cancer prevention and detection among urban medically underserved minority groups. The East Harlem Partnership for Cancer Awareness. Cancer 107:2043-2051. doi:10.1002/cncr. 22153

49. Lisovicz N, Johnson RE, Higginbotham J et al (2006) The Deep South Network for cancer control. Building a community infrastructure to reduce cancer health disparities. Cancer 107:19711979. doi:10.1002/cncr.22151

50. Kagawa-Singer M, Pourat N, Breen N et al (2007) Breast and cervical cancer screening rates of subgroups of Asian American women in California. Med Care Res Rev 64:706-730. doi: $10.1177 / 1077558707304638$

51. Sohn L, Harada ND (2005) Knowledge and use of preventive health practices among Korean women in Los Angeles County. Prev Med 41:167-178. doi:10.1016/j.ypmed.2004.09.039

52. Schootman M, Jeffe DB, Reschke AH, Aft RL (2003) Disparities related to socioeconomic status and access to medical care remain in the United States among women who never had a mammogram. Cancer Causes Control 14:419-425. doi:10.1023/A:10249 41626748

53. Tracy KA, Quillin JM, Wilson DB et al (2008) The impact of family history of breast cancer and cancer death on women's mammography practices and beliefs. Genet Med 10:621-625. doi:10.1097/GIM.0b013e31817c0355

54. Rushton G, Armstrong MP, Gittler J et al (2006) Geocoding in cancer research: a review. Am J Prev Med 30:S16-S24. doi: 10.1016/j.amepre.2005.09.011

55. Krieger N, Chen JT, Waterman PD, Rehkopf DH, Subramanian SV (2005) Painting a truer picture of US socioeconomic and racial/ethnic health inequalities: the Public Health Disparities Geocoding Project. Am J Public Health 95:312-323. doi:10.2105/ AJPH.2003.032482

56. Bonner MR, Han D, Nie J, Rogerson P, Vena JE, Freudenheim JL (2003) Positional accuracy of geocoded addresses in epidemiologic research. Epidemiology 14:408-412

57. Fiscella K, Holt K, Meldrum S, Franks P (2006) Disparities in preventive procedures: comparisons of self-report and medicare claims data. BMC Health Serv Res 6:122. doi:10.1186/14726963-6-122

58. Holt K, Franks P, Meldrum S, Fiscella K (2006) Mammography self-report and mammography claims: racial, ethnic, and socioeconomic discrepancies among elderly women. Med Care 44: 513-518. doi:10.1097/01.mlr.0000215884.81143.da 